\title{
Effects of WT1 down-regulation on oocyte maturation and preimplantation embryo development in pigs
}

\author{
Fei Gao*, Jiyu Guan*, Limei Liu, Sheng Zhang, Peipei An², Anran Fan, Guangqi Song, \\ Peng Zhang, Tianchuang Zhao, Bo Tang, Xueming Zhang and Ziyi Li
}

Jilin Provincial Key Laboratory of Animal Embryo Engineering, College of Veterinary Medicine, Center for Animal Embryo Engineering of Jilin Province, Jilin University, 5333 Xi An Da Lu, Changchun, Jilin 130062, China, ${ }^{1}$ College of Medical Laboratory Science, Beihua University, Jilin, Jilin 132013, China and ${ }^{2}$ Institute of Special Animal and Plant Science, Chinese Academy of Agricultural Sciences, Changchun, Jilin 130122, China

Correspondence should be addressed to Z Li; Email: ziyi@jlu.edu.cn or to be X Zhang; Email: zhxueming@yahoo.com

*(F Gao and J Guan contributed equally to this work)

\begin{abstract}
The Wilms' tumour 1 (WT1) gene originally identified as a tumour suppressor associated with WTs encodes a zinc finger-containing transcription factor that is expressed in multiple tissues and is an important regulator of cellular and organ growth, proliferation, development, migration and survival. However, there is a deficiency of data regarding the expression and function of WT1 during oocyte maturation and preimplantation embryonic development. Herein, we sought to define the expression characteristics and functions of WT1 during oocyte maturation and preimplantation embryonic development in pigs. We show that WT1 is expressed in porcine oocytes and at all preimplantation stages in embryos generated by ICSI. We then evaluated the effects of down-regulating WT1 expression at germinal vesicle and early ICSI stages using a recombinant plasmid (pGLV3-WT1-shRNA). Down-regulation of WT1 did not affect oocyte maturation but significantly decreased preimplantation embryonic development and increased apoptosis in blastocysts. These results indicate that WT1 plays important roles in the development of porcine preimplantation embryos.

Reproduction (2014) 148 377-387
\end{abstract}

\section{Introduction}

The Wilms' tumour (WT1) gene was first identified in 1990 as a tumour suppressor associated with WTs (Call et al. 1990, Gessler et al. 1990). The initial research on the function of the WT1 protein revealed that it is involved in cellular and organ growth, proliferation, development, migration and survival (Algar et al. 1996, Moore et al. 1999). WT1 encodes a zinc finger nuclear transcriptional factor that is expressed during the development of several organs, including the gonads, kidney, spleen and heart (Hohenstein \& Hastie 2006). In adult reproductive organs, WT1 expression has been detected in Sertoli cells of the testes and granulosa cells of ovarian follicles (Buckler et al. 1991). WT1 is also expressed in the nucleoli of Xenopus and marsupial oocytes (Ladomery et al. 2003, Pask et al. 2007). Wt1-deficient mice die pre- or perinatally (Herzer et al. 1999), and the development of the gonadal ridge is abnormal in Wt1-deficient animals (Gao et al. 2005). The importance of $W t 1$ during embryonic development was also demonstrated by the failure of mice with targeted disruptions of $W t 1$ to form kidneys or gonadal tissue (Kreidberg et al. 1993). In situ hybridisation and northern blot studies using human embryos have demonstrated that WT1 is expressed in the differentiating stem cells of the kidney and in the mesonephros, mesothelium and developing gonads. Wt1 is also expressed during the development of tissues in which the mesenchymal-to-epithelial transition occurs (Pritchard-Jones et al. 1990, Armstrong et al. 1993). Based on these findings, it is possible that WT1 might function more broadly than previously thought, indicating a need for detailed information on WT1 expression and function during the development of preimplantation stage embryos.

Recent developmental biology studies have focused on the potential role of $W t 1$ in the regulation of follicular growth. In rats and mice, Wt 1 is expressed in granulosa cells of primordial, primary and secondary follicles, with expression diminishing throughout follicular development (Pelletier et al. 1991, Hsu et al. 1995, Logan et al. 2003). Hsu et al. (1995) reported that one possible role for WT1 is to inhibit the differentiation of immature follicles. Our previous work indicated that downregulation of WT1 directly leads to premature apoptosis 
as well as changes in SF1, SOX9 and GDNF gene expression in porcine kidney fibroblasts and swine testis cells (An et al. 2013).

Although most studies have reported that WT1 is strongly expressed in ovarian follicles and regulates the size of ovary and the number of developing follicles (Logan et al. 2003, Gao et al. 2014), few researchers have investigated its expression characteristics and biological functions during preimplantation embryonic development. Herein, we examined the possibility of an earlier role for $W T 1$ in controlling cell fate decisions using shRNAs that block the WT1 mRNA present during the preimplantation period. We evaluated WT1 mRNA expression and WT1 protein localisation in preimplantation embryos and determined how this gene contributes to early embryonic development. This study thus provides new information on early embryogenesis that is relevant for future developmental biology studies.

\section{Materials and methods}

\section{Chemicals}

All chemicals were purchased from Sigma-Aldrich Chemical Company unless stated otherwise.

\section{Animal care}

All animal experiments in this study were performed in accordance with the guidelines on animal care and use established by the Jilin University Animal Care and Use Committee (Approval ID: 20101008-2).

\section{Oocyte collection and in vitro maturation}

Porcine ovaries were collected at a local commercial abattoir and transported to the laboratory within $2 \mathrm{~h}$ in sterile saline maintained at $35-38.5^{\circ} \mathrm{C}$. The ovaries were washed three times in sterile saline $\left(38.5^{\circ} \mathrm{C}\right)$. Follicular fluid and cumulus oocyte complexes (COCs) were aspirated from 2 to $8 \mathrm{~mm}$ antral follicles with a $10 \mathrm{ml}$ disposable syringe and an 18-gauge needle and expelled into sterile Petri dishes $(9 \mathrm{~cm}$ diameter $)$ kept at $38.5^{\circ} \mathrm{C}$. COCs with more than three layers of intact and compact cumulus cells were selected, washed three times in manipulation fluid (TCM199 supplemented with $0.1 \%$ polyvinyl alcohol) and cultured in in vitro maturation (IVM) media (Liu et al. 2012). A group of $\sim 30$ COCs were cultured in a $100 \mu \mathrm{l}$ drop of maturation media for $42-44 \mathrm{~h}$ at $38.5^{\circ} \mathrm{C}$ in an atmosphere of $5 \% \mathrm{CO}_{2}$ and saturated humidity. All maturation media drops containing oocytes were covered with mineral oil and incubated in pre-equilibrated culture medium.

\section{Spermatozoa preparation}

Fresh semen was supplied by the Jilin University Pig Farm (Changchun, China) and stored at $17^{\circ} \mathrm{C}$. The semen sample was pre-treated using a Percoll gradient. For preparation of 45 and $90 \%$ (vol/vol) Percoll, $2 \mathrm{ml}$ of $45 \%$ Percoll was placed on top of $90 \%$ Percoll in a $12 \mathrm{ml}$ conical centrifuge tube. Then, $0.5 \mathrm{ml}$ of diluted semen were added gently and centrifuged at $700 \mathrm{~g}$ for $30 \mathrm{~min}$ to retrieve live and motile spermatozoa (Matas et al. 2011). Pooled sperm-rich fractions were washed with Tyrode's Albumin Lactate Pyruvate media by centrifugation at $100 \mathrm{~g}$ for $10 \mathrm{~min}$. The second sperm pellet was resuspended in Dulbecco's PBS (DPBS) supplemented with $10 \%$ BSA to give a final concentration of $5 \times 10^{5}$ cells $/ \mathrm{ml}$.

\section{ICSI}

Porcine oocytes were denuded of cumulus cells by repeated pipetting after maturation culture for $44 \mathrm{~h}$. Denuded oocytes were washed three times in supplemented DPBS and transferred to drops of ICSI holding medium (DPBS supplemented with $10 \%$ BSA). Immediately before injection, $5 \mu \mathrm{l}$ of sperm suspension were mixed thoroughly with an equal volume of $10 \%$ polyvinyl pyrrolidone (PVP) solution. Metaphase II (MII) oocytes with a visible first polar body were selected for this experiment. Before ICSI, oocytes were loaded in $5 \mu \mathrm{l}$ microinjection drops placed on a lid of a $100 \times 20 \mathrm{~mm}$ Petri dish (one oocyte per drop). A total of ten micro-drops containing MII oocytes were placed in each lid surrounding central sperm drop with occasional minor modifications. The micro-drops were covered with mineral oil.

Motile spermatozoa with normal morphology were selected from the edge of the sperm-containing droplet. The tail of each spermatozoon was removed by a pulse of Piezo (GarciaRosello et al. 2006a). The head of the spermatozoon was then aspirated into an injection pipette with $8 \mu \mathrm{m}$ inner diameter as described previously (Kurome et al. 2007). A single oocyte was immobilised using a holding pipette and held with its polar body at either the 6 or 12 o'clock position (Garcia-Rosello et al. 2006b). A sperm head was then injected into the cytoplasm at the 3 o'clock position using a Piezo-actuated microinjector (PMM-150FU; Prime Tech, Tsuchiura, Japan) and micromanipulators (MO-102, Narishige, Tokyo, Japan). The temperature was maintained at $38.5^{\circ} \mathrm{C}$ throughout all micromanipulation processes using a heated microscopic stage.

\section{Plasmid construction}

Four different WT1 shRNAs and a negative control shRNA were designed and cloned into the pgpu6/GFP/Neo plasmid (GenePharma Co. Ltd, Shanghai, China) as described previously (An et al. 2013). To assess their knockdown efficiency, the four plasmids were transfected into porcine kidney fibroblasts. At $48 \mathrm{~h}$ after transfection, total RNA was extracted from the cells and the products of the four plasmids were analysed by RT-PCR and using the Gel-Pro Analyzer Software 4.0 (MediaCybernetics, Rockville, MD, USA). The most efficient shRNA was selected and cloned into the pGLV3-H1-GFP-Puro lentiviral vector (GenePharma Co. Ltd). The cloned plasmid was named pGLV3-WT1-shRNA, and pGLV3-Nc-shRNA was used as the control vector.

\section{Perivitelline space injection}

After ICSI, the zygotes were kept in Porcine Zygote Medium 3 (PZM3; Okada et al. 2006). Lentiviral particle injection was 
performed based on a modified perivitelline space injection (PSI) method used to produce transgenic embryos (Tessanne et al. 2012). High-titre lentiviral particles $\left(>1 \times 10^{9}\right.$ infections units $/ \mathrm{ml}$ ) were used to microinject embryos for both the pGLV3-WT1-shRNA group and the pGLV3-Nc-shRNA group. Microinjection of recombinant lentiviral particles beneath the zona pellucida was performed after $3 \mathrm{~h}$ of zygote recovery. PSI was performed with a micropipette, using a steady flow into the perivitelline space for 20-30 s/embryo, thus the perivitelline space could be filled with lentiviral particles. Embryos were then washed three times with PZM3 medium and cultured in the same medium $(20$ embryos $/ 100 \mu \mathrm{l})$ at $38.5^{\circ} \mathrm{C}$ in an atmosphere of $5 \% \mathrm{CO}_{2}$.

\section{Preparation of porcine granulosa cells and cumulus- free oocytes and co-culture of denuded oocytes with granulosa cells}

Cumulus cells of germinal vesicle (GV) stage COCs were removed by gentle pipetting in manipulation media supplemented with $0.1 \%$ hyaluronidase for $15 \mathrm{~min}$. The denuded oocytes were then transferred into maturation media drops, and PSI was performed using the same manipulation described above. After microinjection, the oocytes were washed three times with maturation media and incubated with granulosa cells collected from GV stage oocytes as described previously (Zhang et al. 2010) with minor modifications. The cells were pelleted by centrifugation at $200 \mathrm{~g}$ for $5 \mathrm{~min}$ at room temperature, washed twice in PBS and then resuspended in somatic cell culture medium (DMEM supplemented with $15 \%$ foetal bovine serum) to yield a suspension of $3 \times 10^{5}$ cells $/ \mathrm{ml}$. Then, $1 \mathrm{ml}$ of the cell suspension was plated in each well of a 24-well plastic plate (Corning, One Riverfront Plaza, NY, USA) and cultured at $38.5^{\circ} \mathrm{C}$ in a humidified atmosphere of $5 \% \mathrm{CO}_{2}$. When the granulosa cells grew to $\sim 80 \%$ confluence, the medium was replaced with $1 \mathrm{ml}$ maturation media. After $3 \mathrm{~h}$ of equilibration in a $5 \% \mathrm{CO}_{2}$ incubator, denuded porcine oocytes were placed in a four-well plate (Corning; 80-100 oocytes/well) and co-cultured at $38.5^{\circ} \mathrm{C}$ in $5 \% \mathrm{CO}_{2}$ for $44 \mathrm{~h}$ until the oocytes got to MII stage.

\section{Measurement of intracellular glutathione content}

Intracellular glutathione (GSH) levels were measured with the GSH Assay Kit (Beyotime, The Institute of Biotech, S0052, Shanghai, China) following the manufacturer's instructions.
Each sample was composed of 50 oocytes and three samples were assayed for each treatment. Briefly, denuded oocytes were washed three times in PBS in a $1.5 \mathrm{ml}$ centrifuge tube. Then, $5 \mu \mathrm{l}$ of $0.2 \mathrm{M}$ sodium phosphate buffer containing $10 \mathrm{mM} \mathrm{Na}_{2}$ EDTA $(\mathrm{pH}=7.2)$ and $5 \mu \mathrm{l}$ of $1.25 \mathrm{M}$ phosphoric acid $\left(\mathrm{H}_{3} \mathrm{PO}_{4}\right)$ were added to the samples, which were then vortexed thoroughly, frozen at $-80^{\circ} \mathrm{C}$ and thawed at $37^{\circ} \mathrm{C}$. This procedure was repeated at least three times to lyse the oocytes. The samples were then removed and placed on ice for $5 \mathrm{~min}$, centrifuged at $8000 \mathrm{~g}$ for $10 \mathrm{~min}$ at $4{ }^{\circ} \mathrm{C}$ and stored at $-80^{\circ} \mathrm{C}$. The intracellular content of GSH content was determined by the dithionitrobenzoic acid-glutathione disulphide reductase recycling assay, according to the methods described previously (Bijttebier et al. 2008) with minor modifications. Briefly, $175 \mu \mathrm{l}$ of sodium phosphate buffer containing $0.33 \mathrm{mg} / \mathrm{ml} \mathrm{NADH}$ phosphate, $25 \mu \mathrm{l}$ of $6 \mathrm{mM}$ DTNB0 and $40 \mu \mathrm{l}$ of water were added to each sample tube and mixed. After the mixture was equilibrated at $25{ }^{\circ} \mathrm{C}$ for $15 \mathrm{~min}$, the assay was initiated with the addition of $5 \mu \mathrm{l}$ of $125 \mathrm{IU}$ glutathione disulphide reductase. The absorbance was measured four times at $30 \mathrm{~s}$ intervals at a wavelength of $415 \mathrm{~nm}$ using a spectrophotometer (DU7500; Beckman Coulter, Fullerton, CA, USA). GSH standards and a blank sample lacking GSH were assayed at the same time. The GSH content per oocyte was calculated by dividing the total content per sample by the number of oocytes present in the sample.

\section{RNA isolation, $R T$ and quantitative real-time PCR}

Total RNA was extracted from 50 pre-implantation embryos from the treated and control groups at different developmental stages using the RNeasy Micro Kit (74004, Qiagen) according to the manufacturer's recommendations. cDNA was synthesised using oligo (dT) primers and the PrimeScript First strand cDNA synthesis kit (D6110, TaKara) following the manufacturer's instructions.

mRNA levels were determined using SYBR Green RT-PCR assays. SYBR Premix Ex Taq reagents (DRR041, TaKara, Shiga, Tokyo, Japan) were used for monitoring amplification. Reaction conditions and the temperature profile of the PCR were specified by the manufacturer. Quantitative real-time PCR was performed on an ABI Step Two Real-Time 7500 PCR system (Applied Biosystems). Primers were designed using the online Primer3 Software (Whitehead Institute for Biomedical Research). All primers are given in Table 1. Samples were denatured at $95{ }^{\circ} \mathrm{C}$ for $1 \mathrm{~min}$ and then subjected to 40 cycles of amplification

Table 1 Primers for quantitative real-time PCR.

\begin{tabular}{|c|c|c|c|c|}
\hline Genes & Primer sequences $\left(5^{\prime}-3^{\prime}\right)$ & GenBank accession no. & Annealing temperature $\left({ }^{\circ} \mathrm{C}\right)$ & Length $(\mathrm{bp})$ \\
\hline WT1 & $\begin{array}{l}\text { F: TGAGCGAAGGTTTTCTCGTT } \\
\text { R: GCTGAAGGGCTTTTCACTTG }\end{array}$ & NM_001001264.1 & 65.0 & 166 \\
\hline$B C L 2$ & $\begin{array}{l}\text { F: GAAACCCCTAGTGCCATCAA } \\
\text { R: GGGACGTCAGGTCACTGAAT }\end{array}$ & AJ001203.1 & 60.0 & 196 \\
\hline SOX2 & $\begin{array}{l}\text { F: GCCCTGCAGTACAACTCCAT } \\
\text { R: GCTGATCATGTCCCGTAGGT }\end{array}$ & NM_001123197.1 & 60.0 & 216 \\
\hline POU5F1 & $\begin{array}{l}\text { F: AGTGAGAGGCAACCTGGAGA } \\
\text { R: TCGTTGCGAATAGTCACTGC }\end{array}$ & KJ023672.1 & 60.0 & 166 \\
\hline GAPDH & $\begin{array}{l}\text { F: GTCGGAGTGAACGGATTTG } \\
\text { R: TCTCAGCCTTGACTGTGCC }\end{array}$ & AF017079.1 & 62.0 & 175 \\
\hline
\end{tabular}

$F$, forward primer; $R$, reverse primer. 
$\left(95^{\circ} \mathrm{C}, 5 \mathrm{~s}\right.$ and $\left.65^{\circ} \mathrm{C}, 30 \mathrm{~s}\right)$. Transcript levels were calculated relative to the transcription of the housekeeping gene GAPDH in each sample. Fold changes for every gene were calculated using the $2^{-\Delta \Delta C t}$ method (Livak \& Schmittgen 2001).

\section{Immunofluorescence staining}

Porcine oocytes and embryos with zona pellucida were digested by treatment with $0.5 \%$ Proteinase (Pronase) in PZM3 at $38.5{ }^{\circ} \mathrm{C}$ for $5 \mathrm{~min}$. Zona pellucida-free oocytes and embryos of all groups were fixed with $4 \%$ paraformaldehyde in PBS ( $\mathrm{pH}=7.4$ ) for $30 \mathrm{~min}$, permeabilised for $40 \mathrm{~min}$ with $0.3 \%$ Triton X-100 in PBS and blocked in a blocking solution (PBS supplemented with $3 \%$ BSA) for $30 \mathrm{~min}$ at room temperature. Samples were then incubated with the primary antibody (rabbit polyclonal WT1; 1:200, Abcam (Cambridge, UK), ad96792) diluted in PBS containing 1\% BSA overnight at $4{ }^{\circ} \mathrm{C}$. Oocytes and embryos were then washed three times in PBS containing $0.3 \%$ PVP and transferred into the appropriate secondary antibodies rhodamine-conjugated goat anti-rabbit IgG (1:500, Millipore (Billerica, MA, USA), AP307R) or FITC-conjugated goat anti-rabbit $\operatorname{lgG}(1: 500$, Millipore, $12-507)$ at $37^{\circ} \mathrm{C}$ for $3 \mathrm{~h}$. After washing three times in PBS containing 0.3\% PVP, nuclei were stained with Hoechst $33342(10 \mu \mathrm{g} / \mathrm{ml}$ in PBS) for $10 \mathrm{~min}$. Finally, samples were mounted onto slides and coverslipped with FluorSave. Negative controls were determined by processing samples as described above in the absence of primary antibody. All steps involving fluorescence were carried out in the dark. Samples were observed under a confocal laserscanning microscope (Olympus Fv100). All images were scanned at the same laser power and recorded digitally with a high-resolution CCD camera. At least five embryos at every developmental stage were processed for each experiment, and the experiments were repeated three times.

\section{Apoptosis assays}

TUNEL apoptosis assays were carried out with the fluoresceinconjugated In Situ Cell Death Detection Kit (11684817910, Roche) according to the manufacturer's instructions. After removing zonae pellucidae by treatment with $0.5 \%$ Proteinase in PBS, day 5 blastocysts were fixed in 4\% paraformaldehyde, permeabilised in $0.3 \%$ Triton X-100, washed three times in PBS containing $0.3 \%$ PVP and incubated with the TUNEL solution in the dark for $1 \mathrm{~h}$ at $37^{\circ} \mathrm{C}$. Negative control embryos were incubated with fluorescein-conjugated dUTP. Positive control embryos were incubated in DNase (Roche) for $\sim 20 \mathrm{~min}$ at $37^{\circ} \mathrm{C}$. After TUNEL labelling, the embryos were washed three times in PBS containing $0.3 \%$ PVP. Nuclei were stained for 10 min with Hoechst 33342, and the samples were mounted between a cover slip and a glass slide and examined by confocal microscopy.

\section{Trypan blue viability assay}

To evaluate the lethality phenotype associated with pGLV3WT1-shRNA lentiviral particle microinjection, on day 4, embryos from the WT1-shRNA, Nc-shRNA and non-microinjected groups were incubated in $0.4 \%$ Trypan blue solution diluted 1:9 in PZM3 at room temperature for $10 \mathrm{~min}$. The embryos were then assessed by bright-field microscopy. Blue-stained cells were considered non-viable.

\section{Statistical analysis}

The data were analysed using SPSS (Statistics Production for Service Solution, version 16.0) by one-way ANOVA. Each result is expressed as means \pm s.E.M. of triplicate data obtained from three or more experiments. Comparisons of mean values between control and treated groups were performed using Tukey's multiple comparison tests. Differences with $P<0.05$ or $P<0.01$ were considered statistically significant and extremely significant respectively.

\section{Results}

\section{Expression of WT1 in oocytes and preimplantation embryos}

Temporal and spatial WT1 protein expression patterns were examined in oocytes and preimplantation embryos by immunofluorescence (Fig. 1A). WT1 was expressed in both oocytes and early embryos at all developmental stages and was located in both the nucleus and cytoplasm. Quantitative real-time PCR results showed that in oocytes the WT1 mRNA level was lower at MII stage than at GV stage (Fig. 1B). In preimplantation embryos, WT1 mRNA expression gradually increased from two-cell to morula stages. WT1 mRNA expression was also lower at two-cell stage compared with the eight-cell and morula stages. The WT1 mRNA level peaked at the eight-cell and morula stages and then declined at the blastocyst stage (Fig. 1C).

\section{WT1 down-regulation did not affect oocyte maturation}

The effect of WT1 depletion on early porcine oocyte maturation was performed by introducing WT1-shRNA lentiviral particles into GV stage oocytes. GV stage cumulus-free oocytes were injected into the perivitelline space with one of the two lentiviral particles (pGLV3-WT1-shRNA and pGLV3-Nc-shRNA) and co-cultured with granulosa cells for $44 \mathrm{~h}$. To evaluate the efficiency of the WT1 knockdown, WT1 expression was examined by quantitative real-time PCR. At the MII stage, WT1 expression was decreased in the WT1shRNA-microinjected oocytes compared with control oocytes (Fig. 2A and B). The number of mature oocytes (MII stage) was recorded according to the presence of a visible first polar body (Table 2). Down-regulation of WT1 had no effect on oocyte maturation. GSH levels can also be used as an indicator of cytoplasmic maturation in porcine oocytes (Table 2). We found that GSH levels were not different between oocytes microinjected with pGLV3-WT1-shRNA and controls (non-microinjected and Nc-shRNA). 

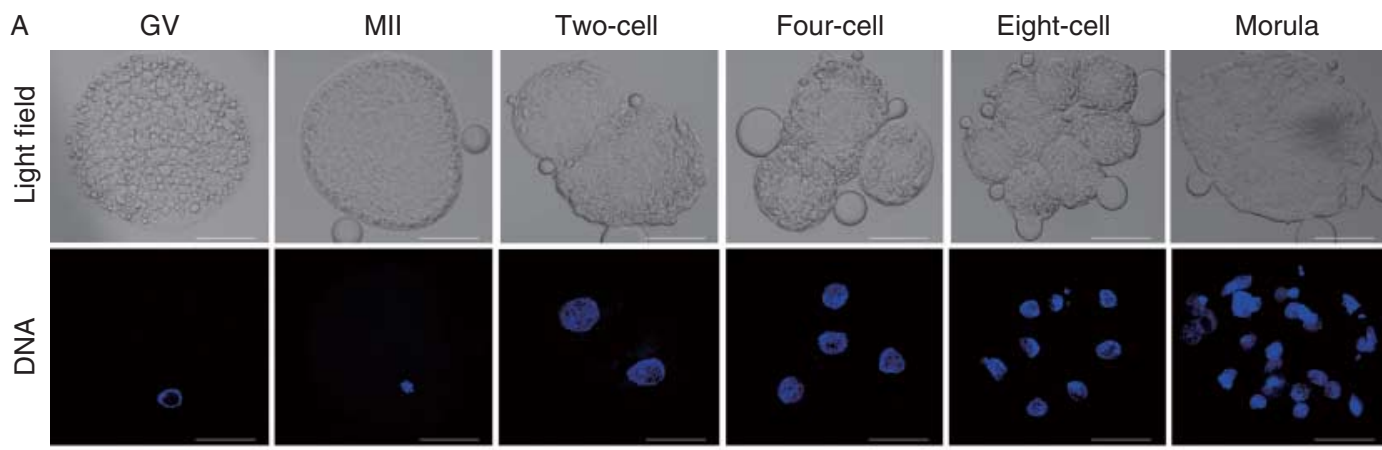

Blastocyst
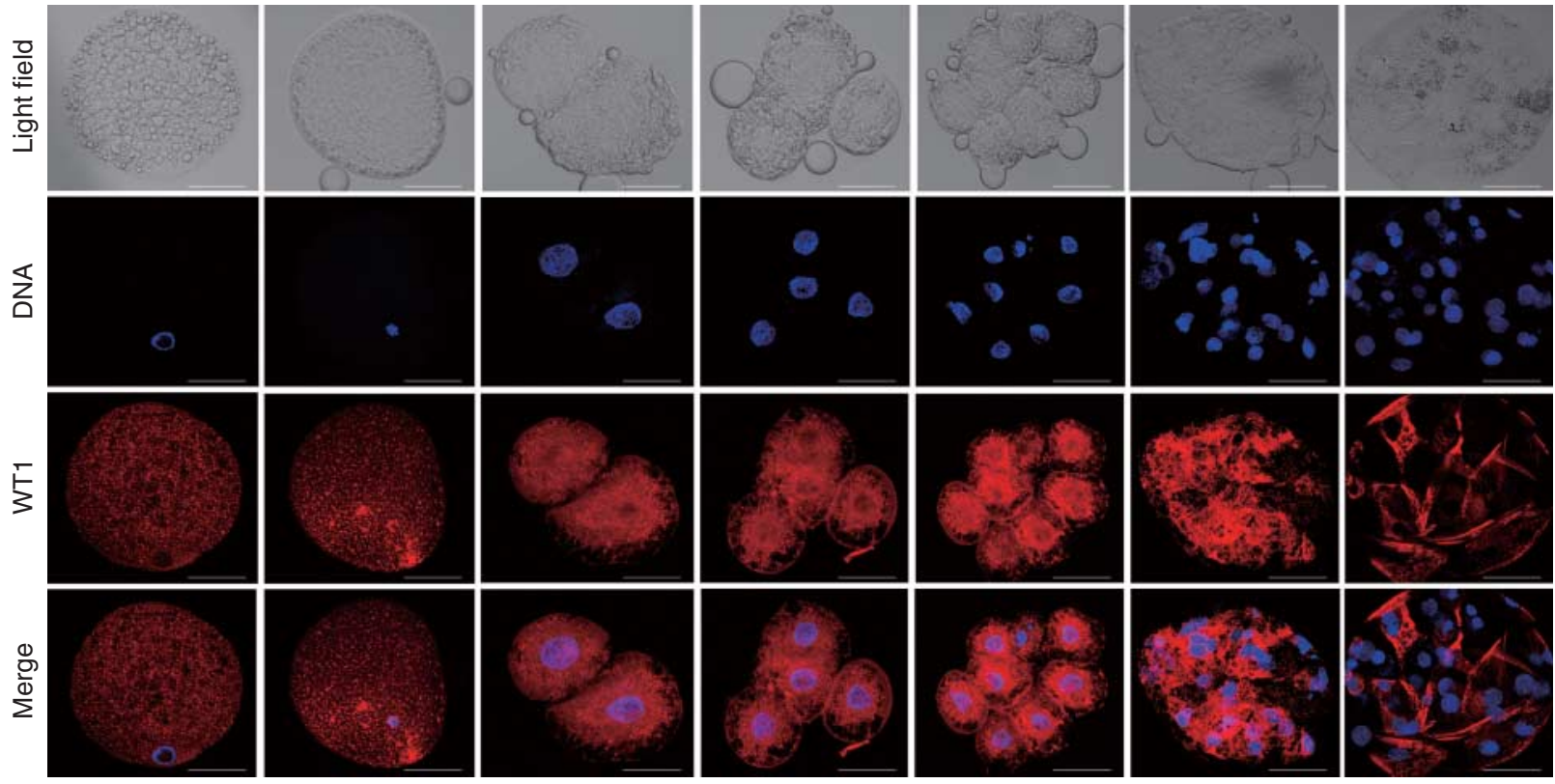

B

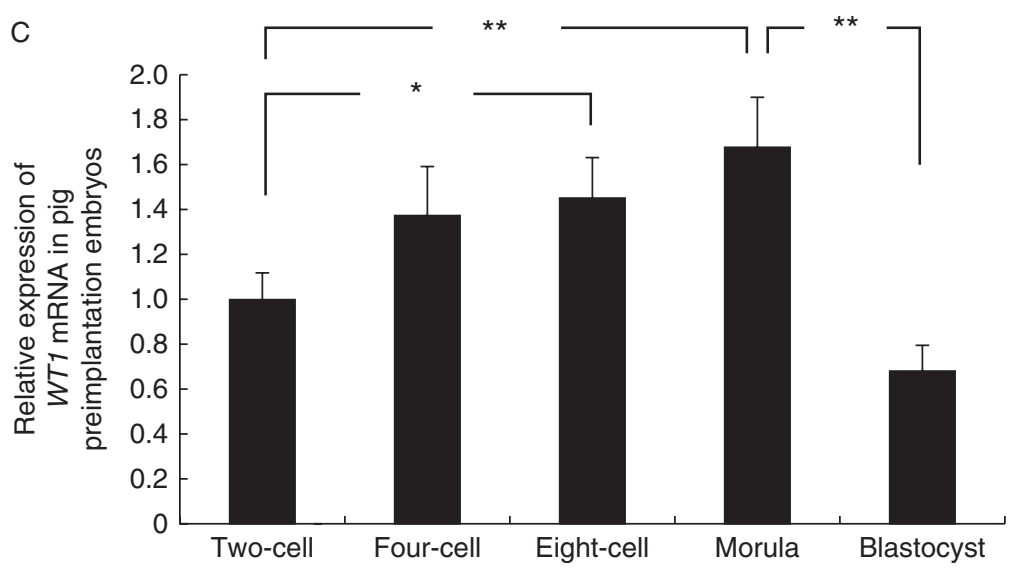

Figure 1 WT1 is expressed in oocytes and preimplantation embryos. Temporal and spatial patterns of $W T 1$ expression during oocyte maturation and all preimplantation stages were evaluated by immunofluorescence staining and quantitative real-time PCR. (A) WT1 protein immunofluorescence in oocytes and preimplantation embryos revealed by a WT1 antibody (red); each sample was counterstained with Hoechst 33342 to visualise DNA (blue). Scale bar: $50 \mu \mathrm{m}$. (B and C) Quantitative real-time PCR for WT1 in oocytes and all preimplantation developmental stages, using GAPDH as the control. The data are expressed as means \pm s.D. from three independent experiments. ${ }^{*} P<0.05$ and ${ }^{* *} P<0.01$.

\section{WT1 down-regulation in zygotes leads to early embryonic arrest}

To further evaluate the function of WT1 in porcine preimplantation embryonic development, pGLV3-WT1shRNA was microinjected into zygotes. The embryos were cultured and collected on days 1 and 5, at the twocell and blastocyst stages respectively. The per cent cleavage of in vitro cultured embryos on day 1 did not differ between the control and treated groups (Fig. 3A). On day 5, 59.2 and $56.5 \%$ of the ICSI embryos from the control and Nc-shRNA groups had reached the blastocyst stage respectively. However, in the WT1-shRNA lentiviral particle-microinjected group, only $9.2 \%$ of the embryos had reached the blastocyst stage by day 5
$(P<0.01$ compared with controls; Fig. 3B and C). Most of the WT1-shRNA lentiviral particle-microinjected embryos were arrested at the eight-cell $(28.0 \%)$ or morula stage $(32.2 \%)$. By contrast, embryos in the nonmicroinjected and Nc-shRNA-microinjected control groups were arrested at the eight-cell stage at rates of 7.6 and $11.6 \%$, respectively, and at the morula stage at rates of 5.5 and $5.0 \%$ (Fig. 3C). A Trypan blue (T6146, Sigma-Aldrich) uptake assay was performed on day 4 embryos to examine the lethality phenotype associated with WT1-shRNA lentiviral particle microinjection (Fig. 3D and E). Approximately $84 \%$ of the WT1shRNA lentiviral particle-microinjected embryos contained some necrotic (blue-stained) cells. 
A
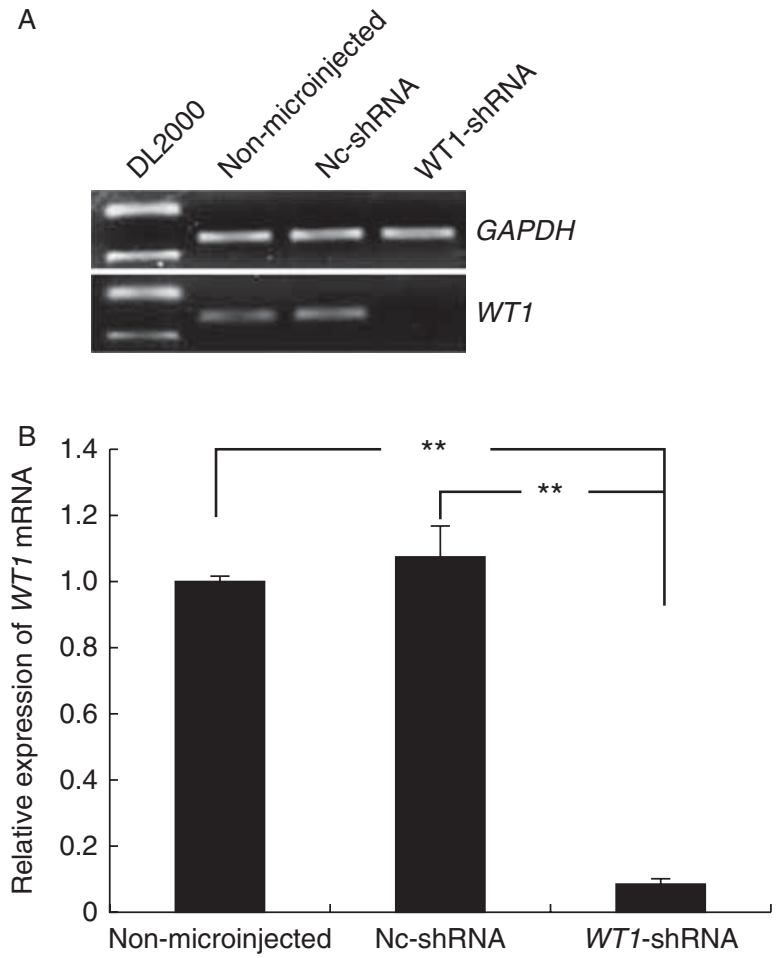

Figure 2 The effects of $W T 1$-shRNA lentiviral particle microinjection on WT1 expression in MII oocytes. (A) WT1 mRNA expression in MIl oocytes as assessed by conventional RT-PCR using GAPDH as a control. (Lane 1) DL2000 DNA marker (D501A, Takara). (B) Quantitative real-time PCR was used to measure WT1 expression in MII oocytes. The data are expressed as means \pm s.D. from three independent experiments. ${ }^{* *} P<0.01$.

WT1 mRNA and protein levels were assessed by quantitative real-time PCR and immunofluorescence at each preimplantation embryonic stage. WT1 transcript levels were measured by quantitative real-time PCR on preimplantation embryos from all groups. Compared with non-microinjected and Nc-shRNA lentiviral particle-microinjected groups, WT1 levels in the WT1shRNA lentiviral particle-microinjected group were decreased (Fig. 4A and B). To confirm the aforementioned results, WT1 protein level was examined by immunofluorescence staining. Both the non-microinjected and the Nc-shRNA lentiviral particle-microinjected control groups showed strong staining for WT1, particularly at the eight-cell and morula stages (Fig. 4C). By contrast, the embryos in the WT1-shRNA lentiviral particle-microinjected group showed extremely faint WT1 staining that continued until the blastocyst stage (Fig. 4C). Porcine kidney fibroblasts were used as a positive control for WT1 immunofluorescence staining (Supplementary Fig. 1A, see section on supplementary data given at the end of this article); preimplantation embryos of all developmental stages that only stained with a secondary antibody were used as negative controls (Supplementary Fig. 1B).

\section{WT1 down-regulation induced apoptosis in preimplantation embryos}

To determine whether WT1 knockdown influenced ICSI embryonic development, which is primarily reflected by blastocyst quality, the number of apoptotic cells was estimated by a TUNEL assay performed on day 5 . As shown in Fig. $5 \mathrm{~A}$ and $\mathrm{B}$, the number of apoptotic cells in WT1-shRNA blastocysts was higher than that of nonmicroinjected and Nc-shRNA lentiviral particle-microinjected embryos. However, in a morphology comparison, the total number of cells per blastocyst did not differ among the three groups (Fig. 5C).

Anti-apoptotic gene $B C L 2$ mRNA level was examined using quantitative real-time PCR at various developmental stages among non-microinjected, Nc-shRNA and WT1-shRNA lentiviral particle-microinjected groups. The WT1-shRNA lentiviral particle-microinjected group showed a lower BCL2 mRNA level at every developmental stage than controls (non-microinjected and Nc-shRNA; Fig. 5D).

\section{Discussion}

The WT1 gene first identified in 1990 encodes a zinc finger protein that functions as a tumour suppressor and plays important roles in the development of the urogenital system and several other tissues (Call et al. 1990, Gessler et al. 1990, Rivera \& Haber 2005). WT1 may also play a role in follicular growth (Logan et al. 2003). We previously found that WT1 is essential for the development and survival of porcine kidney fibroblasts and testis cells, as its down-regulation increased apoptosis and affected the expression of several survival genes (An et al. 2013). Herein, we further report that WT1 is also expressed in oocytes and preimplantation embryos, suggesting a potential important function in early embryonic development.

Previous studies have suggested that WT1 is primarily localised to the nucleus in cancer cells (Menssen et al. 2000, Caballero et al. 2001). However, Silberstein et al. (1997) noted that WT1 is distributed in the cytoplasm but not nuclei in some human breast cancers and suggested that such localisation may be regulated by alternative

Table 2 Effect of WT1-shRNA on cytoplasmic maturation and intracellular glutathione (GSH) levels in in vitro-matured porcine oocytes.

\begin{tabular}{lccc}
\hline IVM & $\begin{array}{c}\text { Number of } \\
\text { oocytes used }^{\text {a }}\end{array}$ & $\begin{array}{c}\text { Percentage } \\
\text { of oocytes } \\
\text { reached MII }\end{array}$ & $\begin{array}{c}\text { GSH level } \\
\left(n=50^{\mathrm{b}}\right)\end{array}$ \\
\hline Non-microinjected & 241 & $91.91 \pm 1.79^{*}$ & $7.78 \pm 0.50^{*}$ \\
NC-shRNA & 205 & $90.04 \pm 5.35^{*}$ & $7.56 \pm 0.48^{*}$ \\
WT1-shRNA & 196 & $89.32 \pm 1.73^{*}$ & $7.51 \pm 0.32^{*}$ \\
\hline
\end{tabular}

The data are expressed as means \pm S.E.M. In the same column, differences among the numbers containing the same symbols in the superscripts were not statistically significant $(P>0.05)$.

${ }^{\mathrm{a}}$ Four replicates. ${ }^{\mathrm{b}}$ Number of embryos examined. Four replicates. 

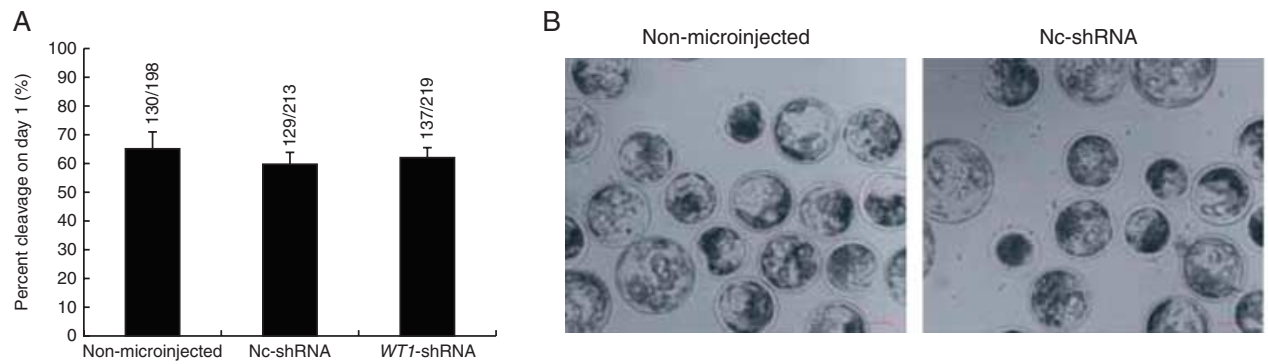

D Non-microinjected

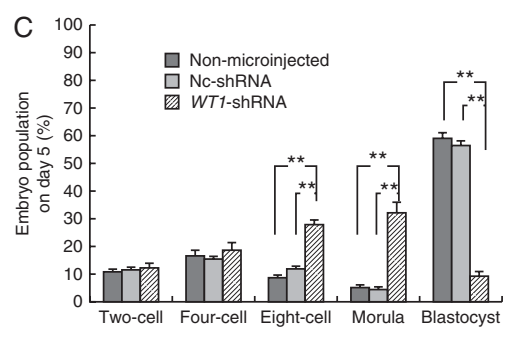

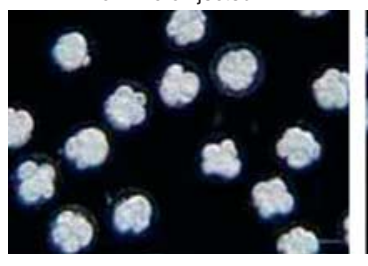

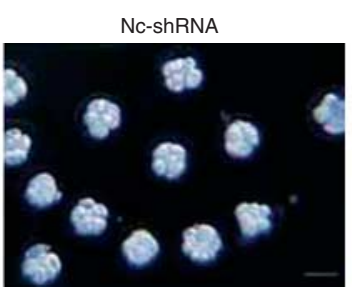

WT1-shRNA
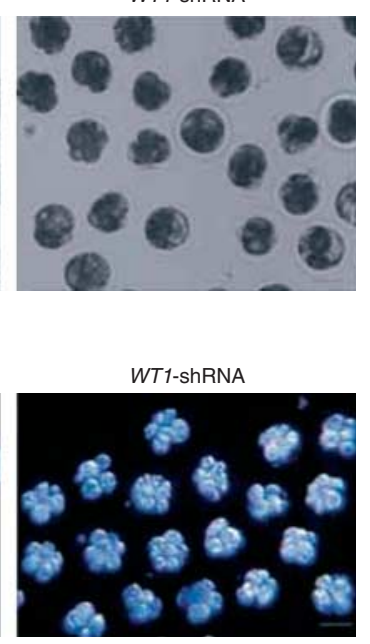

E

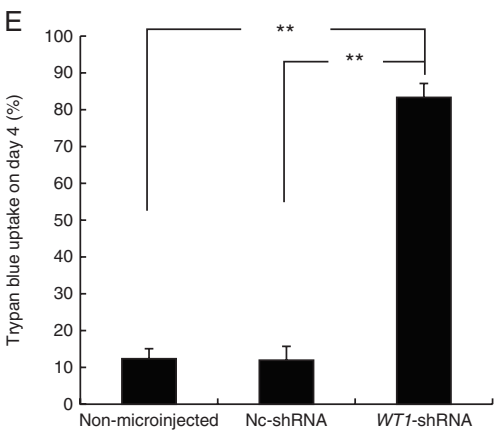

Figure 3 Assessment of cleavage and development of early embryos. (A) WT1-shRNA lentiviral particle microinjection had no effect on the cleavage of preimplantation embryos on day 1; (B) WT1-shRNA lentiviral particle microinjection blocked the development of preimplantation embryos. Phase-contrast images of non-microinjected, Nc-shRNA and WT1-shRNA lentiviral particle-microinjected embryos on day 5. Scale bars: $100 \mu$ m; (C) Percentages of embryos at the indicated stages in the three groups after culture to day 5; (D) Trypan blue uptake assay for preimplantation embryos. Presence of non-viable (blue) cells in WT1-shRNA lentiviral particle-microinjected embryos at day 4. Scale bars: $100 \mu \mathrm{m}$; (E) Percentage of non-microinjected, Nc-shRNA and WT1-shRNA lentiviral particle-microinjected embryos showing Trypan blue uptake on day 4. Values are expressed as mean \pm s.D. from three independent experiments. ${ }^{*} P<0.01$.

splicing of WT1 mRNA. Extensive evidence has accumulated indicating that WT1 acts as a complex, multifunctional nuclear protein involved in at least two levels of gene expression regulation: RNA transcription and processing (Niksic et al. 2004). WT1 has also been shown to undergo nucleocytoplasmic shuttling (Vajjhala et al. 2003), as predicted by Ye et al. (1996). Researchers have also reported that WT1 may be retained in the cytoplasm after being phosphorylated, thereby preventing nuclear events related to DNA binding and RNA transcription (Takahara et al. 2011). Our results showed that WT1 protein was expressed in porcine oocytes and early ICSI embryos at all developmental stages examined and was located in both the nucleus and cytoplasm.

In ovaries, both granular and epithelial cells show high levels of WT1 expression (Pelletier et al. 1991). Logan et al. (2003) reported that in antral follicles, WT1 may prevent premature differentiation of somatic follicle cells during early follicular growth. WT1 is also expressed in the nucleolus in Xenopus and marsupial oocytes
(Ladomery et al. 2003, Pask et al. 2007). We thus speculated that WT1 would be expressed in porcine oocytes and might play an important role in porcine oocyte maturation. Our results from immunofluorescence and quantitative real-time PCR analysis in oocytes indicate that WT1 mRNA and protein are expressed at both the GV and MII stages. We then evaluated WT1 function by infecting GV stage oocytes with pGLV3WT1-shRNA lentiviral particles and examining oocyte quality and rate of maturation. Oocyte quality is one of the most critical factors determining viability in vitro and developmental competence in in vitro-produced embryos. GSH is produced in porcine oocytes during the maturation process (Yoshida et al. 1993), and intraoocyte GSH levels are considered an important indicator of cytoplasmic maturation. Interestingly, we found no difference between the treated and control groups in either oocyte maturation rates or GSH levels in IVM porcine oocytes. We observed that WT1-down-regulated oocytes were able to undergo normal maturation, reach 
A

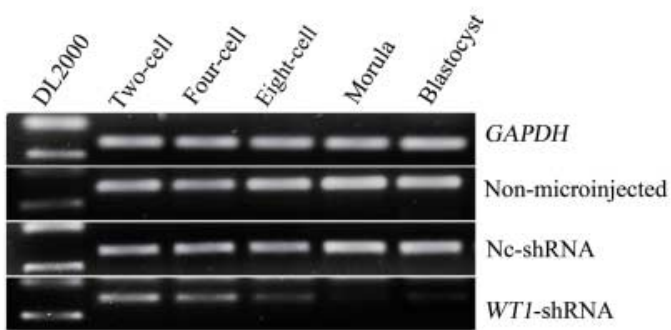

B

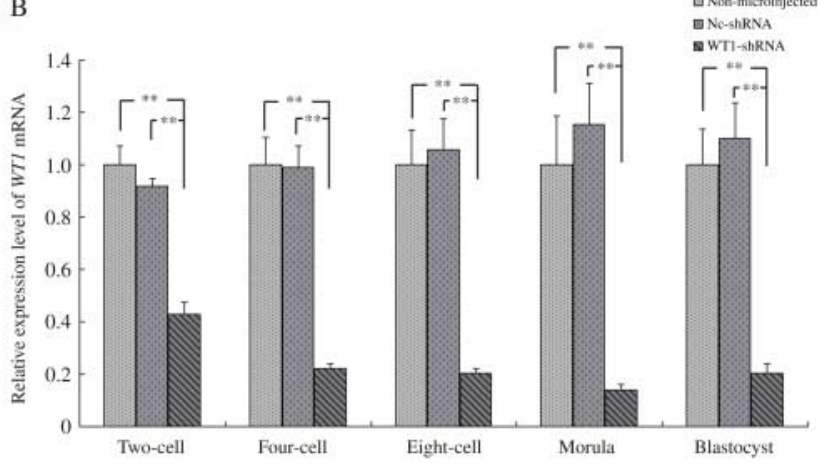

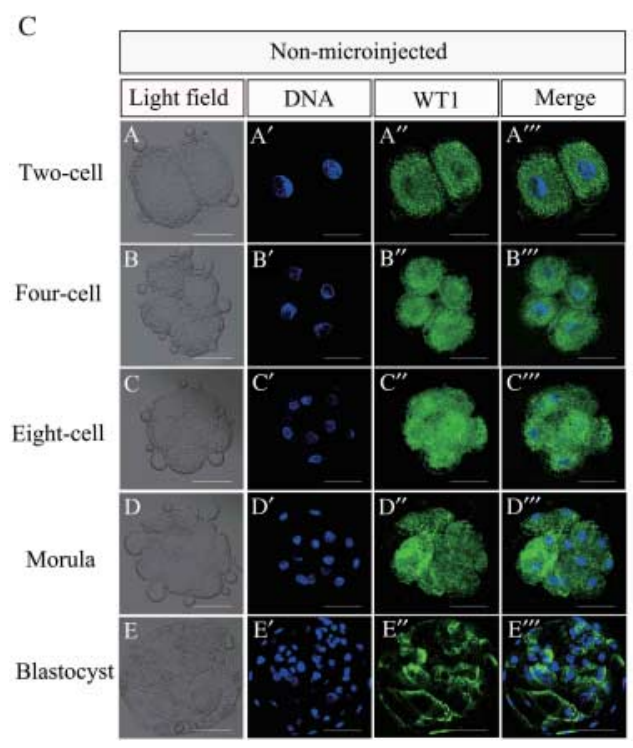
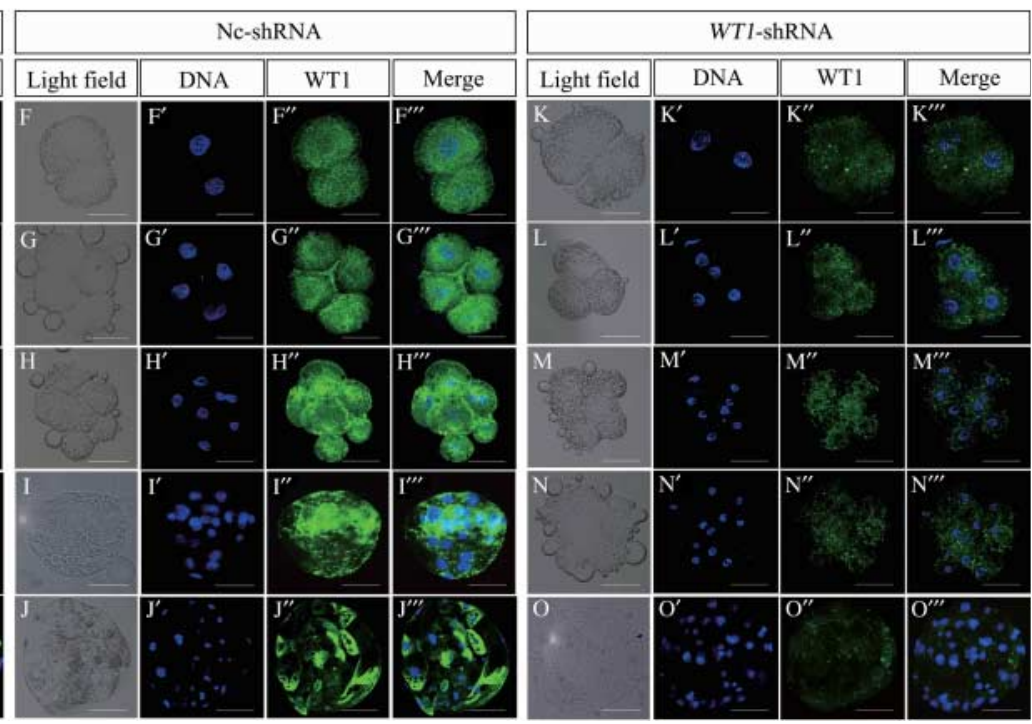

Figure 4 WT1 expression at all preimplantation developmental stages in non-microinjected, Nc-shRNA and WT1-shRNA lentiviral particlemicroinjected embryos. (A) WT1 mRNA expression was assessed by conventional RT-PCR using GAPDH as a control; (B) relative expression of WT1 mRNA in preimplantation embryos as assessed by quantitative real-time PCR and (C) the effects of WT1-shRNA lentiviral particle microinjection on WT1 expression at preimplantation stages. Confocal images of control non-microinjected, control Nc-shRNA lentiviral particle-microinjected and WT1-shRNA lentiviral particle-microinjected embryos at every preimplantation developmental stage. Embryos were immunostained with a WT1 antibody (green), and the DNA was counterstained with Hoechst 33342 (blue). Scale bar: $50 \mu \mathrm{m}$. Each value represents mean \pm s.D. from three independent experiments. ${ }^{* *} P<0.01$.

the MII stage from the GV stage and emit the first polar body. Thus, there are no indications that pGLV3-WT1shRNA lentivirus-mediated down-regulation of WT1 mRNA in porcine oocytes has any effect on oocyte maturation.

It has been reported that the rate of development to the blastocyst stage of IVF embryos is higher than that of ICSI embryos; however, the quality of IVF-derived blastocysts is not as good as that of ICSI-derived blastocysts (Park et al. 2005). Li et al. (2013) reported that IVF might cause polyspermy, which can result in elevated expression of the proapoptosis gene $B A X$ and loss of the anti-apoptosis gene $B C L 2 L 1$ ( $B C L X L)$ in blastocysts. Given these factors, we chose to use ICSI embryos for our study. shRNA targeting gene technology has been widely used in scientific studies, including many related to livestock improvement (Long et al. 2010). Herein, we constructed a pGLV3-WT1-shRNA lentiviral vector (An et al. 2013) and used it to infect oocytes and ICSI preimplantation embryos. Most of the WT1-down-regulated embryos were arrested at the eight-cell or morula stages, though some were able to develop into blastocysts. These embryos showed lower levels of WT1 protein expression than observed in control groups. These findings indicated that the WT1 down-regulation was incomplete in these embryos, such that enough WT1 protein had accumulated by the eight-cell and morula stages to allow them to develop into blastocysts. To further determine the role of WT1 in eight-cell- and morulastage embryos, the Trypan blue method was used to examine the lethality phenotype of the WT1-shRNA group on day 4 . Herein, we found that the blastomere became necrotic in a majority of the embryos. To address the specificity of WT1 knockdown, two developmentrelated genes SOX2 and POU5F1 (OCT4) mRNA levels have been examined. We have found that at all 


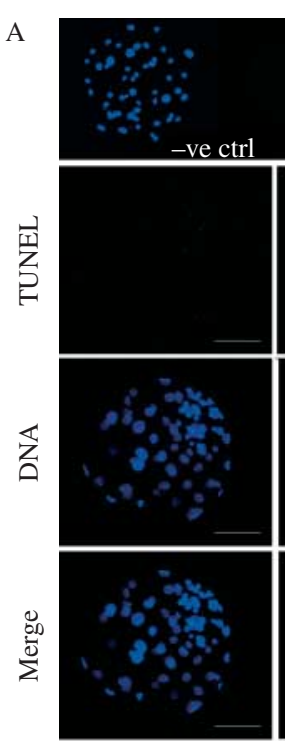

Non-microinjected
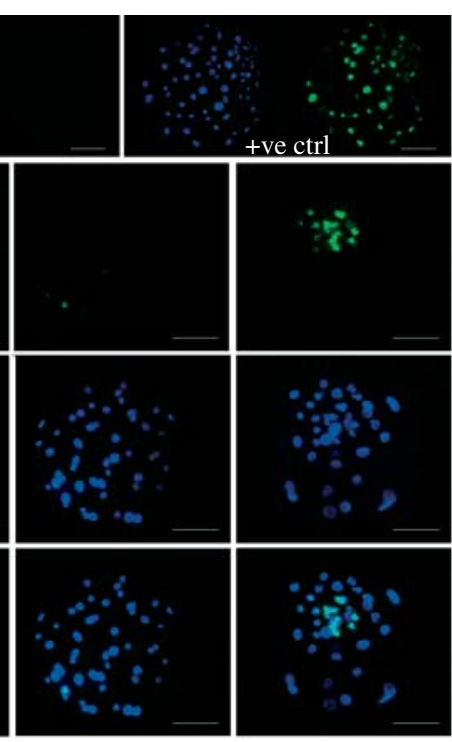

Nc-shRNA

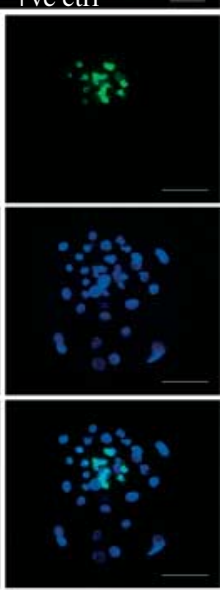

WT1-shRNA
B

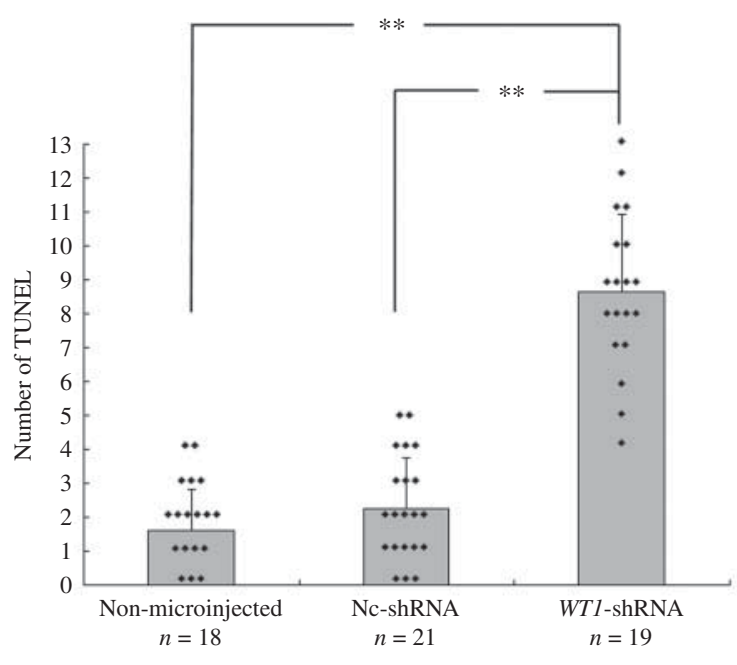

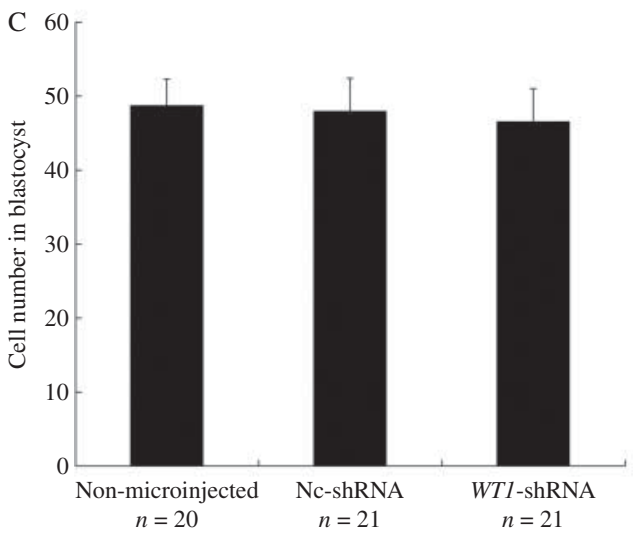

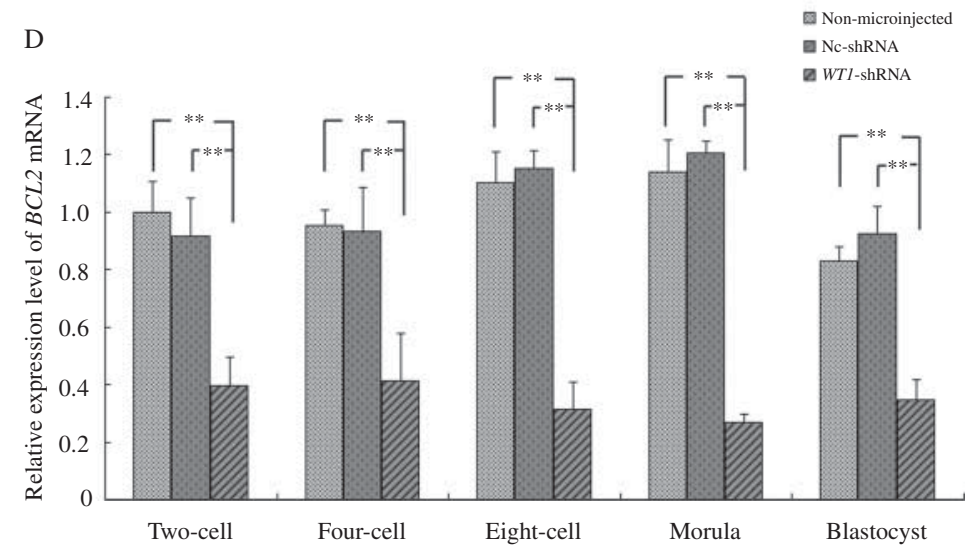

Figure 5 Assessment of apoptosis in WT1-down-regulated embryos. (A) TUNEL assay on blastocysts. The fragmented nuclei (green) are apoptotic; Controls for the TUNEL reaction ( - ve ctrl is negative control and + ve ctrl is positive control). Each sample was counterstained with Hoechst 33342 to visualise DNA (blue). Scale bar: $50 \mu \mathrm{m}$. (B) Number of apoptotic cells in each group. * is one blastocyst. The data are expressed as means \pm S.E.M. ${ }^{* *} P<0.01 ; n=18-21$. (C) Cell number per blastocyst in WT1-shRNA lentiviral particle-microinjected and control groups. The data are expressed as means \pm s.E.M. $n=20-21$. (D) Quantitative real-time PCR for BCL2 at each developmental stage between WT1-shRNA lentiviral particlemicroinjected and control groups. The results shown are expressed as means \pm s.D.s from three independent experiments. $* * P<0.01$.

preimplantation stages, SOX2 and POU5F1 mRNA expression level have no difference between WT1 down-regulation group and the control groups, which may minimise the possibility of the off-target effects (Supplementary Fig. 2A and B, see section on supplementary data given at the end of this article).

Infection with pGLV3-WT1-shRNA particles dramatically decreased WT1 expression at both the mRNA and protein levels. A small number of the WT1-downregulated embryos developed into blastocysts, though apoptosis assays showed that the number of apoptotic cells was increased in these embryos compared with controls. The lentiviral particle infection itself did not appear to increase apoptosis, and all ICSI embryos exhibited low levels of apoptosis. Therefore, the increased apoptosis observed in the WT1-downregulated embryos seems to be a specific effect. These findings indicate that down-regulation of WT1 might activate an apoptotic pathway. BCL2 is an anti-apoptotic protein (Kirkin et al. 2004). Mayo et al. (1999) reported that WT1 has an anti-apoptotic function that may be related to increase $B C L 2$ levels. Therefore, we examined the expression of $B C L 2$ in this study. $B C L 2$, as an antiapoptotic gene, has also been reported as a potential target of WT1 (Loeb 2006) and it is expressed in oocytes and embryos (Abazari-Kia et al. 2014). We have found that the level of anti-apoptotic gene BCL2 was downregulated at every preimplantation stage of WT1 downregulated group, which suggests that the apoptosis induced by WT1 knockdown is mediated through 
$B C L 2$ down-regulation. We have also previously found that $B C L 2$ levels decreased as $B A X$ levels increased in WT1-knockdown porcine testis cells (An et al. 2013).

In this study, we report that WT1 mRNA and protein are present in porcine oocytes and at all preimplantation stages in ICSI embryos. Upon down-regulation of WT1, most early ICSI embryos were arrested at the eight-cell or morula stage. A small number of these embryos developed into blastocysts, though they presented a dramatically increased number of apoptotic cells. We thus reveal a major role for $W T 1$ in preimplantation embryos that are essential for early embryonic development.

\section{Supplementary data}

This is linked to the online version of the paper at http://dx.doi. org/10.1530/REP-14-0204.

\section{Declaration of interest}

The authors declare that there is no conflict of interest that could be perceived as prejudicing the impartiality of the research reported.

\section{Funding}

This work was supported by the National Natural Science Foundation (no. 31072027), the National Basic Research Program (no. 2011CB944204), Postdoctoral Special Funded Projects (no. 201003536) and the Program for Changjiang Scholars and Innovative Research Team in University (no. IRT1248) in China.

\section{References}

Abazari-Kia AH, Mohammadi-Sangcheshmeh A, DehghaniMohammadabadi M, Jamshidi-Adegani F, Veshkini A, Zhandi $M$, Cinar MU \& Salehi M 2014 Intracellular glutathione content, developmental competence and expression of apoptosis-related genes associated with G6PDH-activity in goat oocyte. Journal of Assisted Reproduction and Genetics 31 313-321. (doi:10.1007/s10815-0130159-y)

Algar EM, Khromykh T, Smith SI, Blackburn DM, Bryson GJ \& Smith PJ 1996 A WT1 antisense oligonucleotide inhibits proliferation and induces apoptosis in myeloid leukaemia cell lines. Oncogene 12 1005-1014.

An P, Yin Y, Fan A, Tan W, Gao F, Ma K, Tang B, Zhang X \& Li Z 2013 The effects of the WT1 gene on apoptosis and development-related gene expression in porcine kidney fibroblasts and swine testis cells. Molecular Reproduction and Development 80 414-425. (doi:10.1002/mrd.22176)

Armstrong JF, Pritchard-Jones K, Bickmore WA, Hastie ND \& Bard JB 1993 The expression of the Wilms' tumour gene, WT1, in the developing mammalian embryo. Mechanisms of Development 40 85-97. (doi:10. 1016/0925-4773(93)90090-K)

Bijttebier J, Van Soom A, Meyer E, Mateusen B \& Maes D 2008 Preovulatory follicular fluid during in vitro maturation decreases polyspermic fertilization of cumulus-intact porcine oocytes in vitro maturation of porcine oocytes. Theriogenology 70 715-724. (doi:10. 1016/j.theriogenology.2008.04.046)

Buckler AJ, Pelletier J, Haber DA, Glaser T \& Housman DE 1991 Isolation, characterization, and expression of the murine Wilms' tumor gene (WT1) during kidney development. Molecular and Cellular Biology 11 1707-1712. (doi:0270-7306/91/031707-06\$02.00/0)
Caballero OL, de Souza SJ, Brentani RR \& Simpson AJ 2001 Alternative spliced transcripts as cancer markers. Disease Markers 17 67-75. (doi:10.1155/2001/184856)

Call KM, Glaser T, Ito CY, Buckler AJ, Pelletier J, Haber DA, Rose EA, Kral A, Yeger H, Lewis WH et al. 1990 Isolation and characterization of a zinc finger polypeptide gene at the human chromosome 11 Wilms' tumor locus. Cell 60 509-520. (doi:10.1016/0092-8674(90)90601-A)

Gao X, Chen X, Taglienti M, Rumballe B, Little MH \& Kreidberg JA 2005 Angioblast-mesenchyme induction of early kidney development is mediated by Wt1 and Vegfa. Development 132 5437-5449. (doi:10. 1242/dev.02095)

Gao F, Zhang J, Wang X, Yang J, Chen D, Huff V \& Liu YX 2014 Wt1 functions in ovarian follicle development by regulating granulosa cell differentiation. Human Molecular Genetics 23 333-341. (doi:10.1093/ $\mathrm{hmg} / \mathrm{ddt} 423$ )

Garcia-Rosello E, Matas C, Canovas S, Moreira PN, Gadea J \& Coy P 2006 a Influence of sperm pretreatment on the efficiency of intracytoplasmic sperm injection in pigs. Journal of Andrology 27 268-275. (doi:10.2164/ jandrol.05110)

Garcia-Rosello E, Coy P, Garcia Vazquez FA, Ruiz S \& Matas C $2006 \mathrm{~b}$ Analysis of different factors influencing the intracytoplasmic sperm injection (ICSI) yield in pigs. Theriogenology 66 1857-1865. (doi:10. 1016/j.theriogenology.2006.04.037)

Gessler M, Poustka A, Cavenee W, Neve RL, Orkin SH \& Bruns GA 1990 Homozygous deletion in Wilms tumours of a zinc-finger gene identified by chromosome jumping. Nature 343 774-778. (doi:10.1038/343774a0)

Herzer U, Crocoll A, Barton D, Howells N \& Englert C 1999 The Wilms tumor suppressor gene wt1 is required for development of the spleen. Current Biology 9 837-840. (doi:10.1016/S0960-9822(99)80369-8)

Hohenstein P \& Hastie ND 2006 The many facets of the Wilms' tumour gene, WT1. Human Molecular Genetics 15 R196-R201. (doi:10.1093/ hmg/ddl196)

Hsu SY, Kubo M, Chun SY, Haluska FG, Housman DE \& Hsueh AJ 1995 Wilms' tumor protein WT1 as an ovarian transcription factor: decreases in expression during follicle development and repression of inhibin- $\alpha$ gene promoter. Molecular Endocrinology 9 1356-1366. (doi:10.1210/ mend.9.10.8544844)

Kirkin V, Joos S \& Zornig M 2004 The role of Bcl-2 family members in tumorigenesis. Biochimica et Biophysica Acta 1644 229-249. (doi:10. 1016/j.bbamcr.2003.08.009)

Kreidberg JA, Sariola H, Loring JM, Maeda M, Pelletier J, Housman D \& Jaenisch R 1993 WT-1 is required for early kidney development. Cell $7 \mathbf{4}$ 679-691. (doi:10.1016/0092-8674(93)90515-R)

Kurome M, Saito H, Tomii R, Ueno S, Hiruma K \& Nagashima H 2007 Effects of sperm pretreatment on efficiency of ICSI-mediated gene transfer in pigs. Journal of Reproduction and Development 53 1217-1226. (doi:10.1262/jrd.19069)

Ladomery M, Sommerville J, Woolner S, Slight J \& Hastie N 2003 Expression in Xenopus oocytes shows that WT1 binds transcripts in vivo, with a central role for zinc finger one. Journal of Cell Science $\mathbf{1 1 6}$ 1539-1549. (doi:10.1242/jcs.00324)

Li XX, Lee DS, Kim KJ, Lee JH, Kim EY, Park JY \& Kim MK 2013 Leptin and nonessential amino acids enhance porcine preimplantation embryo development in vitro by intracytoplasmic sperm injection. Theriogenology 79 291-298. (doi:10.1016/j.theriogenology.2012.08.019)

Liu L, Song G, Gao F, Guan J, Tang B \& Li Z 2012 Transient exposure to sodium butyrate after germinal vesicle breakdown improves meiosis but not developmental competence in pig oocytes. Cell Biology International 36 483-490. (doi:10.1042/CBI20110220)

Livak KJ \& Schmittgen TD 2001 Analysis of relative gene expression data using real-time quantitative PCR and the $2(-$ Delta Delta $C(\mathrm{~T}))$ method. Methods 25 402-408. (doi:10.1006/meth.2001.1262)

Loeb DM 2006 WT1 influences apoptosis through transcriptional regulation of Bcl-2 family members. Cell Cycle 5 1249-1253. (doi:10.4161/cc.5. 12.2807)

Logan KA, McNatty KP \& Juengel JL 2003 Expression of Wilms' tumor gene and protein localization during ovarian formation and follicular development in sheep. Biology of Reproduction 68 635-643. (doi:10. 1095/biolreprod.102.008946)

Long CR, Tessanne KJ \& Golding MC 2010 Applications of RNA interference-based gene silencing in animal agriculture. Reproduction, Fertility, and Development 22 47-58. (doi:10.1071/RD09211) 
Matas C, Vieira L, Garcia-Vazquez FA, Aviles-Lopez K, Lopez-Ubeda R, Carvajal JA \& Gadea J 2011 Effects of centrifugation through three different discontinuous Percoll gradients on boar sperm function. Animal Reproduction Science 127 62-72. (doi:10.1016/j.anireprosci.2011.06.009)

Mayo MW, Wang CY, Drouin SS, Madrid LV, Marshall AF, Reed JC, Weissman BE \& Baldwin AS 1999 WT1 modulates apoptosis by transcriptionally upregulating the bcl-2 proto-oncogene. EMBO Journal 18 3990-4003. (doi:10.1093/emboj/18.14.3990)

Menssen HD, Bertelmann E, Bartelt S, Schmidt RA, Pecher G, Schramm K \& Thiel E 2000 Wilms' tumor gene (WT1) expression in lung cancer, colon cancer and glioblastoma cell lines compared to freshly isolated tumor specimens. Journal of Cancer Research and Clinical Oncology 126 226-232. (doi:10.1007/s004320050037)

Moore AW, McInnes L, Kreidberg J, Hastie ND \& Schedl A 1999 YAC complementation shows a requirement for Wt1 in the development of epicardium, adrenal gland and throughout nephrogenesis. Development 126 1845-1857.

Niksic M, Slight J, Sanford JR, Caceres JF \& Hastie ND 2004 The Wilms' tumour protein (WT1) shuttles between nucleus and cytoplasm and is present in functional polysomes. Human Molecular Genetics 13 463-471. (doi:10.1093/hmg/ddh040)

Okada K, Krylov V, Kren R \& Fulka J Jr 2006 Development of pig embryos after electro-activation and in vitro fertilization in PZM-3 or PZM supplemented with fetal bovine serum. Journal of Reproduction and Development 52 91-98. (doi:10.1262/jrd.17059)

Park CY, Uhm SJ, Song SJ, Kim KS, Hong SB, Chung KS, Park C \& Lee HT 2005 Increase of ICSI efficiency with hyaluronic acid binding sperm for low aneuploidy frequency in pig. Theriogenology 64 1158-1169. (doi:10.1016/j.theriogenology.2005.01.010)

Pask AJ, Paplinska JZ, Shaw G, Graves JA \& Renfree MB 2007 Marsupial WT1 has a novel isoform and is expressed in both somatic and germ cells in the developing ovary and testis. Sexual Development 1 169-180. (doi:10.1159/000102106)

Pelletier J, Schalling M, Buckler AJ, Rogers A, Haber DA \& Housman D 1991 Expression of the Wilms' tumor gene WT1 in the murine urogenital system. Genes and Development 5 1345-1356. (doi:10.1101/gad.5.8. 1345)

Pritchard-Jones K, Fleming S, Davidson D, Bickmore W, Porteous D, Gosden C, Bard J, Buckler A, Pelletier J, Housman D et al. 1990 The candidate Wilms' tumour gene is involved in genitourinary development. Nature 346 194-197. (doi:10.1038/346194a0)
Rivera MN \& Haber DA 2005 Wilms' tumour: connecting tumorigenesis and organ development in the kidney. Nature Reviews. Cancer $\mathbf{5}$ 699-712. (doi:10.1038/nrc1696)

Silberstein GB, Van Horn K, Strickland P, Roberts CT Jr \& Daniel CW 1997 Altered expression of the WT1 Wilms tumor suppressor gene in human breast cancer. PNAS 94 8132-8137. (doi:10.1073/pnas.94.15.8132)

Takahara A, Koido S, Ito M, Nagasaki E, Sagawa $Y$, Iwamoto T, Komita H, Ochi T, Fujiwara H, Yasukawa M et al. 2011 Gemcitabine enhances Wilms' tumor gene WT1 expression and sensitizes human pancreatic cancer cells with WT1-specific T-cell-mediated antitumor immune response. Cancer Immunology and Immunotherapy 60 1289-1297. (doi:10.1007/s00262-011-1033-3)

Tessanne K, Golding MC, Long CR, Peoples MD, Hannon G \& Westhusin ME 2012 Production of transgenic calves expressing an shRNA targeting myostatin. Molecular Reproduction and Development 79 176-185. (doi:10.1002/mrd.22007)

Vajjhala PR, Macmillan E, Gonda T \& Little M 2003 The Wilms' tumour suppressor protein, WT1, undergoes CRM1-independent nucleocytoplasmic shuttling. FEBS Letters 554 143-148. (doi:10.1016/S00145793(03)01144-X)

Ye Y, Raychaudhuri B, Gurney A, Campbell CE \& Williams BR 1996 Regulation of WT1 by phosphorylation: inhibition of DNA binding, alteration of transcriptional activity and cellular translocation. EMBO Journal 15 5606-5615.

Yoshida M, Ishigaki K, Nagai T, Chikyu M \& Pursel VG 1993 Glutathione concentration during maturation and after fertilization in pig oocytes: relevance to the ability of oocytes to form male pronucleus. Biology of Reproduction 49 89-94. (doi:10.1095/biolreprod49.1.89)

Zhang X, Miao Y, Zhao JG, Spate L, Bennett MW, Murphy CN, Schatten H \& Prather RS 2010 Porcine oocytes denuded before maturation can develop to the blastocyst stage if provided a cumulous cell-derived coculture system. Journal of Animal Science 88 2604-2610. (doi:10. 2527/jas.2009-2714)

Received 15 April 2014

First decision 30 May 2014

Revised manuscript received 10 July 2014

Accepted 15 July 2014 